\title{
L'homme intégral : dignité humaine et transcendance
}

\section{Grégory Woimbée}

Lorsque Mgr Philippe Bordeyne, Recteur de cette vénérable université et le Père Olivier Artus, Vice-Recteur à la Recherche, m'ont fait l'honneur excessif de m'inviter à vous parler à l'occasion de la rentrée doctorale, mes recherches du moment me poussèrent à donner un titre fort suggestif, mais si vaste que je risquais de m'y noyer. Je prie l'auditoire de ne pas voir dans ce qui va suivre un traité ou une leçon académique, mais plutôt un essai qui tente d'articuler quelques réflexions personnelles dont je voudrais qu'elles ne vous semblent pas superflues ou qu'elles vous donnent à penser, manière pour moi de vous renvoyer la balle, car tout doctorant devrait se poser la question de la vision de l'homme qui entre dans sa recherche et qui lui sert de matrice, tout comme celle qu'elle dessine pour lui et ses futurs lecteurs ou collaborateurs de recherche. Le chercheur n'entend pas s'adresser à la seule académie, il entend parler à tout le monde, le grand public, parce qu'il travaille pour le bien commun. Lorsqu'il cherche au sein d'une université catholique, il ajoute à ses destinataires un troisième public, l'Eglise, relié aux deux autres par le fait de sa propre identité académique, sociale et ecclésiale ${ }^{1}$.

J'ai voulu faire le lien entre des convictions qui sont nées de ma propre experience de chercheur, dix années me séparant de ma soutenance de thèse - le 7 octobre 2007 - à l'université grégorienne sur la raison théologique chez Bernard Lonergan ${ }^{2}$. Entre temps, il m'a fallu enseigner la christologie $\mathrm{i}^{3}$, la théologie fondamentale ${ }^{4}$ ou encore partager mes premières amours : l'oeuvre splendide de Romano Guardini ${ }^{5}$. C'est l'avantage d'être bercé par les grands que de se savoir constamment petit ou indigne, nain sur les épaules d'un géant ${ }^{6}$. Et l'avantage d'être contraint à l'humilité, trop pénible à atteindre autrement, c'est d'avoir accès à des sphères et des représentations qui font ignorer les vaines polémiques d'écoles, de courants ou de confessions. La théologie de controverse a sclérosé la pensée chrétienne pendant des décennies, rétrécissant ici, amalgamant là. Elle a apauvrit ce qu'elle a voulu défendre, croyant à tort que c'était la vérité toute pure que ses contemporains rejetaient et non le vinaigre dont elle la recouvrait.

«Intégral» est un mot qui au sens le plus commun désigne l'unité que forme un tout composé et dont chaque dimension n'est pas une partie mais un aspect, si bien que le retrait de l'un n'entraine pas seulement l'amputation, mais la disparition pure et simple de la réalité ainsi composée. « Intégral » renvoie à l'impératif d'union contre le risque d'une disparition, en l'occurrence du substantif auquel il est ici accolé : l’homme. Mais n'est-ce pas redondant de dire «homme intégral » dans la mesure où l'homme est intégral par nature? La redondance est utile lorsqu'elle appuie là où cela fait mal. L'homme, intégral par nature, n'est plus intégral dans les conceptions anthropologiques dominantes, dualistes spiritualistes ou monistes matérialistes qui ont plutôt pour effet de le désintégrer. Trop souvent cerné par des definitions abstraites, l'homme avec un grand $\mathrm{H}$ a servi à exclure des pans considérables de l'humanité. Plus l'homme était conscient de lui-même, plus sa definition s'affinait par

\footnotetext{
${ }^{1}$ Cf. TraCY, David, The analogical Imagination. Christian Theology and the Culture of Pluralism, New York, Crossroad, p.XI : «Nous avons besoin de concevoir une stratégie théologique nouvelle et nécessairement complexe, pour éviter la privatisation de la religion en articulant sa prétention première à la vérité. » (Nous traduisons de l'anglais). A partir de la différenciation des trois sphères publiques (société et théologie fondamentale, académie et théologie systématique, Eglise et théologie pratique) et le constat d'une privatisation et d'une marginalisation de la théologie, Tracy propose une systématique herméneutique en construisant une analogie entre la signification et la vérité de la religion à partir de la signification et de la vérité de l'art. L'analogie entre art et religion lui permet de produire (par ce qu'il appelle analogical imagination)une théorie non classique des classiques (donc de conserver les classiques tout en sortant de leur interprétation classique).

2 Cf. La presqu'île du divin. Objectivité de la raison théologique chez. Bernard Lonergan, Paris, Cerf, La nuit surveillée, 2010.

${ }^{3}$ Cf. Lecons sur le Christ. Introduction à la christologie, Perpignan, Artège, Sed Contra, 2013.

${ }^{4}$ Cf. Leçons sur la foi. Introduction à la théologie fondamentale, Paris/Perpignan, Artège/Lethielleux, 2016.

${ }^{5}$ Cf. L'Esprit du Christianisme. Introduction à la pensée de Romano Guardini, Genève, Ad Solem, 2008.

${ }^{6}$ « Nani gigantum humeris insidentes », métaphore attribuée à Bernard de Chartres (XII e siècle).
} 
les sciences de la nature, plus petite était la part des hommes traités comme tels ou des hommes responsables de leurs actes en vue d'un bien dépassant l'utilité ou l'intérêt d'un pouvoir ascendant ou dominant, à croire que les privilèges accordés à la definition étaient retirés à la foule des hommes concrets. Cet élitisme idéologique n'est pas récent, mais il s'est doublé, sous couvert d'universalisme, d'un élitisme technologique. Il a fait bougé ses lignes au cours du temps, mais sans jamais remettre réellement en cause le principe d'une démarcation effective entre les hommes. L'expression « homme intégral » entend dire qu'un homme n'est homme, et à reconnaître ainsi comme détenteur des droits et obligations de l'humanité, que si sa dignité ne dépend pas de lui, et donc que si sa definition lui échappe. Le terme désigne donc l'homme comme «totalité » (unité composée) et comme "dignité instrinsèque ", ce qui signifie que sa liberté ne peut venir d'une pure déclaration du sujet (autonomie), mais d'une loi qui le transcende et qu'il ne peut se donner à lui-même (hétéronomie) s'il ne l'a pas reçue comme telle à partir d'un Être transcendant qui la lui révèle comme modalité de son être, à la fois grâce et destinée (théonomie participée).

On pourra voir dans ce qui suit un ensemble décousu entre un terminus quo sur l'intelligence chrétienne et un terminus ad quem sur la dignité humaine. Délié en apparence, car une réflexion sur l'homme intégral peut faire le lien entre l'acte par lequel l'homme comprend et se comprend et la comprehension qu'il peut atteindre de sa dignité et du lien de celle-ci au Dieu qui lui parle et qu'il prie. J'essaierai de cerner cet homme intégral en trois temps et de l'appréhender successivement en quête de vérité (1), en devenir de Dieu (2) et en impératif de dignité (3).

\section{I - L'homme en quête de vérité}

Vous le savez, même si la recherche doctorale a souvent l'air d'une conquête, la vérité n'est jamais le résulat d'une conquête, mais d'un recueillement. Le recueillement lui-même ne se conquiert pas. Il est plutôt l'effet d'une mystérieuse gratuité en soi à laquelle on participe. En revanche, il n'y aurait aucune thèse sans qu'ait été conquis le conquérant qui sommeille en son auteur, lequel agit trop souvent sur son objet en ignorant tout ce qui agit sur lui, jusqu'à l'objet lui-même.

La tâche du chercheur en théologie fondamentale que je suis est d'aller du centre aux périphéries, ex corde fidei - à la fois par un acte humain qui prend sa source en Dieu et par un contenu révélé à des hommes choisis, reçu et transmis par des Christi fideles en communion synchronique et diachronique les uns avec les autres - ad laborem mundi, là où sont les hommes, tous les hommes, croyants ou non, confessants ou non, pratiquants ou non. Et pour accomplir cette tâche, il faut faire la route en sens inverse, aller des hommes à la Parole que Dieu leur adresse. Celui qui prétendrait partir directement du centre se contenterait de l'illusion d'être là où il n'est pas, où nul ne se trouve, à commencer par Dieu lui-même, et, par consequent, de déverser son trop plein de doctrine sur des nécessiteux et des ignorants. Il faut aller de l'un à l'autre et inversement, si l'on ne veut pas finir l'un contre l'autre, ou pire encore, l'un sans l'autre 7 . La voie ascendante est le corrolaire de la voix descendante et non son contraire. Et cette voie ascendante n'est pas la raison seule; s'y mêle tout l'ordre des significations prélinguistiques, prévolitives et préréflexives, là où l'imagination est une puissance régalienne, là où l'art et le symbole sont des villes ouvertes à parcourir, d'autant que cet ordre ne disparait pas lorsque la grâce divine est donnée ou qu'on agit par elle. La raison, pure ou pratique, aurait bien tort de s'en passer ou deviendrait folle rien qu'à essayer de le faire. Cette voie présume ce qui la rend possible, elle se guide à sa voix et le cherche en ses profondeurs. Opposer, comme il est tentant et facile de le faire la confession la plus pure aux confessants les plus fragiles, théologie d'une part et anthropologie de l'autre, doctrine d'une part et pastorale de l'autre, revient toujours à appauvrir le message chrétien, à le caricaturer, voir même à le voiler au benefice de l'athéisme ${ }^{8}$.

A l'heure où, dans l'Eglise, certains tenants du centre et de la périphérie s'opposent, le théologien peut offrir la preuve qu'ont tort en même temps ceux qui campent intra muros et ceux qui s'exilent hors-les-murs. Ces murs n'existent pas, n'ont jamais existé, nos bastions sont des chimères, que nous voulions les abattre ou nous en faire un refuge. Les grands auteurs chrétiens sont de précieux

\footnotetext{
${ }^{7}$ Cf. Jean-Paul II, enc. Fides et Ratio, 1998, n.67.

${ }^{8}$ Cf. Concile Vatican II, Const. Past. Gaudium et Spes, 1965, n.19.
} 
exemples parce qu'ils commencent toujours par fuir les idéologies, ces pensées toutes faites incontroversables, puis par les combattre autant lorsqu'elles viennent du dehors que lorsqu'elles se forment au dedans, en privilegiant sans cesse l'acte de compréhension et la question qui l'exprime. Dans notre rapport à la vérité, qu'il s'agisse de celle qu'on atteint ou reçoit par ses propres forces, la vérité de notre compréhension de ce qui est, notre sens du réel ici-bas, ou de celle à laquelle Dieu nous ouvre et nous conduit, l'offre salvifique, notre questionnement est illimité tandis que nos réponses sont limitées. Opposer des idées toutes faites à des idées toutes faites n'est pas d'un grand secours, tandis que réapprendre à penser, hors des systèmes et sans esprit de système, est une autre affaire, noble, d'une grandeur qui nécessite l'humilité, celle de Dieu selon la chair, celle de l'homme selon l'esprit. La pensée n'est pas une école. C'est plutôt son acte de transmission ou l'enseignement qui forgent des générations d'enseignants et d'étudiants. La théologie d'école ne pense pas, mais elle enseigne du mieux possible ce qui a été pensé, contribuant ainsi à établir des dispositions à l'acte de penser par soi-même chez l'élève, et non à lui dire ce qu'il faut penser. La méthode est la clef: dire, non pas ce qu'il faut penser, mais comment l'on pense, et le découvrir en pensant, in actu exercito.

Si la recherche de la vérité doit guider l'acte de penser et toute démarche intellectuelle, il faut savoir de quoi on parle. Pour chercher la vérité, il faut chercher à l'atteindre, et donc à la désirer, et surtout la désirer comme quelque chose qu'on ne doit pas vouloir posséder. La posséder, c'est la fabriquer. L'opinion n'est pas la vérité, et rien n'est grave dans l'opinion sauf à l'ignorer. Cette relation à la vérité qu'on veut atteindre sans vouloir la posséder procède d'une relation personnelle d'oblation. Le chercheur avance en se donnant soi-même. Et ce type de relation cognitive, et quasi amoureuse puisqu'elle n'est mue que par l'amitié qui fait renoncer à la possession, ne dure que dans la permanence du don personnel initial qui la fonde. L'intelligence la plus pointue, si elle n'est pas le fait d'une humanité ouverte, de comportements civils, pleins de bonté, et surtout d'un progrès spirituel ou d'une dilatation intérieure - certains parleraient d'un dynamisme de conversion intellectuelle et morale - peut être la pire des choses, même si, par ailleurs, cette humanité ouverte ne suffit pas à la rendre pointue.

Je voudrais, après ce preambule qui situe personnellement tout chercheur, aborder le point d'une préoccupation mienne, et sans doute que beaucoup partagent : celle du poids de la théologie, plus largement ad extra - hors des frontières chrétiennes, considérées au sens d'une chrétienté ou d'un christinianisme constitué, non pas seulement comme doctrine ou dépôt immuable de la foi, mais comme moment intellectuel, politique et culturel de l'histoire chrétienne - de la contribution chrétienne à la compréhension et donc aussi aux orientations que l'humanité donne à la présence et à la destinée humaines, dans un contexte de profondes mutations anthropologiques, et plus spécifiquement ad intraau sein de l'Eglise, non pas seulement considérée comme hiérarchie ou magistère, mais comme communauté de personnes en communion spirituelle et sociale bic et nunc en vertu et en vue d'une communion divine incohative - de la contribution de l'intelligence chrétienne à la crise ecclésiale dont on ne peut nier qu'elle a de profondes racines intellectuelles. Le risque est que l'intelligence chrétienne perde en significativité et devienne insignifiante, parce qu'inaudible et constituée comme un bloc qui vient a posteriori juger (ou, beaucoup mieux, lui donner des critères de discernement) ce qui vient ou apparaît, sans contribuer à l'émergence de ce qui apparaît. Le discernement est inséparable d'une offre positive de sens, d'une proposition, d'une alternative crédible, désirable et engageante, nous dirions aujourd'hui - mais le mot est galvaudé - innovante. L'Eglise, comme « lieu où fleurit l'Esprit » est par nature un terrain d'innovations incroyablement fertiles. Tout y pousse à condition que le grain soit bon et qu'il soit semé. Il me semble que la perte de la dimension sapientielle de l'intelligence chrétienne est l'une des causes de la perte de sa fonction matricielle dans la pensée humaine. Loin de moi l'idée de promouvoir une seule seconde une quelconque sécularisation du discours chrétien ou adaptation paresseuse de ses grands enseignements à l'air du temps.

Je suis frappé, cependant, par les débats internes concernant l'exhortation apostolique postsynodale Amoris Laetitia, par la veine tentative de l'opposer à l'enseignement de l'encyclique Splendor Veritatis - une solide étude historique et rédactionnelle montrerait sans difficulté le contraire - pour imposer des positions rigides qui n'ont jamais été canonisées par l'Eglise, et certainement pas par l'encyclique Splendor Veritatis. Je suis preoccupé par la montée d'un catholicisme "identitaire", dont 
l'identité promue néglige tout ce qu'implique la dimension théologale de la vie chrétienne ou lui fait absorber anhistoriquement toutes les dimensions de la vie humaine, niant ainsi l'autonomie du biologique, du psychologique, du politique, du social, etc... Le christianisme n'est pas d'abord une identité à défendre, il est un message à transmettre fondé sur un témoignage, c'est-à-dire sur l'exemple d'une vie transformée, d'une personne liberée et d'une joie partagée. La mélancolie s'empare de celui qui observe le monde à partir du journal télévisé, mais sa foi, ainsi mise à nue, demeure une force qui lui permet d'assumer toute faiblesse, non pour en faire une force, mais pour en faire un chemin de conversion, c'est-à-dire un passage à l'amour le plus vrai qui soit, le plus indemne, non de souffrance mais de haine. Pas plus que le doute ne s'oppose à la foi - c'est la peur - la souffrance ne s'oppose à l'amour : c'est encore et toujours la peur qui s'oppose à nous, en nous et par nous. La victoire sur la peur est l'horizon d'une vie et donc aussi de toute recherche féconde.

L'acte de foi, comme accueil de la vérite divine et conversion intellectuelle, morale et religieuse du chercheur entre encore plus nécessairement et intensément chez le théologien. Mais nous savons que toute science part de prémisses qu'elle ne démontre pas et dépend de dispositions intérieures du chercheur qu'elle ne décrète pas. Un bon théologien est d'abord un honnête homme et cela vaut pour le philosophe, l'historien ou le physicien. L'idée d'une supériorité morale de tel ou tel corromprait la moralité même de l'acte de pensée qu'il met en jeu. La morale n'est pas posée en amont comme un paravent ou un préliminaire, mais plutôt en aval comme une visée et un fruit. Pour le comprendre, nos précédesseurs sont d'inestimables trésors. L'antériorité intellectuelle n'est pas une supériorité morale, mais seul un point tangible d'aboutissement nous permet de constituer un nouveau point de départ.

Ce recul historique - comme sens de l'histoire qui n'est pas la projection d'un système clos de pensée ou d'une idéologie mais l'art de savoir d'où on vient et ce qu'on doit à ceux qui, derrière nous, sont passés avant nous - doit nous éviter la tyrannie du passé, forgée sur l'idée d'un passé immobile et fixe qui n'existe plus et qui n'a même jamais existé tel que l'imagine le passéiste, mais de lui préférer l'idée d'une mémoire une, ordonnée et objectivée. Le sens de l'histoire, si nécessaire aux études, est une mémoire objectivée et le passé dont elle se nourrit fait de nous des héritiers, non pas nostalgiques, mais débiteurs, non pas tant à l'égard des générations précédentes qu'à celui de nos enfants et petits-enfants. Nos prédécesseurs nous aident à les former parce qu'ils nous ont transmis. C'est pour eux, demain, que nous cherchons aujourd'hui, que nous prenons tant de peine à lire, à écrire, à comprendre, à juger, à décider. L'exercice de la pensée, comme épreuve de cohérence et renouvellement, est au service de celui qui n'est pas encore né. Cependant, le refus d'un sens passéiste n'est pas la recherche d'un sens futuriste, mais d'un sens qui s'appuie sur le passé comme sur sa propre expérience, qui ne s'invente pas ex nibilo, mais qui offre le moyen de continuer sa course à travers des significations nouvelles, c'est-àdire des voies nouvelles d'accès au sens originel, qui nous le font atteindre à nouveau. J'œuvre aujourd'hui pour que le sens m'atteigne, mais surtout pour qu'il atteigne un jour celui qui va naitre aujourd'hui et demain, car avec le sens vient le goût et avec le goût vient la marche.

\section{II - L'homme en devenir de Dieu}

Qu'ont en commun nos recherches respectives sur le plan épistémologique, sur le plan des principes qui régissent le savoir organisé par la connaissance ? Parmi beaucoup d'autres choses, d'entendre forger une connaissance herméneutique. Nous interprétons ce que nous lisons. Nous lisons beaucoup, nous interprétons tout le temps, et le plus souvent des interprétations d'interprétations usque ad infinitum. Et pour conduire notre exégèse, nous utilisons des principes, nous engageons notre propre subjectivité, c'est-à-dire que nous nous confrontons constamment à cet autrui qui n'est pas là, et non pas seulement aux objets inanimés qui sont là. Nous travaillons sur les significations humaines, nous produisons à partir d'elles et, dans la rencontre avec elles, nous en établissons d'autres. Nous recueillons du mieux possible des données et leurs interprétations successives dans l'histoire, nous repérons les nœuds dialectiques, tout ce qui relie et oppose ces interprétations successives, nous optons nousmêmes pour un propos sur elle, à la manière du juge qui opte à partir d'une jurisprudence, d'une série autorisée et sélectionnée de jugements antérieurs, non pas à propos de ces points de vue antérieurs, 
mais à propos d'un cas concret et contemporain sur lequel il faut trancher. Nous ne sommes pas arbitres des élégances, ce qui est en général peu élégant sur le plan intellectuel et fort indélicat sur le plan moral, mais nous parlons du présent et de l'avenir. Autrement dit, nous prenons position, avec tout l'étayage intersubjectif nécessaire, après des heures de travail, de réflexions, des errements et changements d'avis sans nombre, des phases de grand brouillard, des moments allant du doute à la certitude, des remises en cause, des abattements succédant aux grandes espérances, en tout en collaboration avec des compagnons d'infortune ou de fortune selon les jours. Prendre position, non pour affirmer notre point de vue sur le monde, mais pour lui offrir, ainsi qu'à soi-même, une meilleure compréhension de lui-même, un meilleur accès au sens coextensif à son être. Si l'herméneute désigne le sens, c'est parce qu'il cherche l'être, c'est qu'il est convaincu qu'il y a du sens attaché à ce qui est, que le monde n'est pas immédiat à lui-même, et que la conscience ou la subjectivité humaine le prouvent constamment. S'il s'intéresse aux mots, c'est en vue du réel dont ils sont le grain.

La vérité est dynamique, mais pas seulement, elle est également symphonique. En effet, la signification n'est pas seulement affaire de linguistique, et la langue n'est pas seulement affaire de logique. Le problème de la vérité n’est pas limité à celui du discours : il est d'abord esthétique, symbolique, éthique et interpersonnel. Si l'homme ne sait ce qu'il est ou plutôt qui il est sans savoir d'où il vient et où il va, sans la découverte d'un sens qu'il exprime par des significations qui y donnent concrètement accès, l'homme ne sait pas non plus qui il est sans faire l'expérience des médiations par lesquelles il est en rapport avec le monde. Là où émerge la signification, elle remplit aussi une fonction que présupposent l'idée d'un développement historique de l'homme et la possibilité d'une lecture de cette histoire humaine. Ecrire son histoire, c'est identifier des variations de la signification, que seul un invariant peut saisir : la différence n'apparaît que lorsqu'est fixée une identité qui s'aperçoit qu'elle change. L'homme est à la fois une substance et un sujet historique, il devient, il apprend, il change, il expérimente qu'il change. Le sens lui apparait ainsi. L'intégralité de l'expérience humaine a du sens, en premier lieu tout un ensemble d'expériences prévolitives et préréflexives, les plus puissants vecteurs de sens par lesquels l'homme sort de l'immédiateté sont l'intersubjectivité, l'art ou l'esthétique, le symbolique. Le sens linguistique et conceptuel se déploie à partir d'eux et non l'inverse. Il n'a pas de sens en soi, et surtout pour moi, s'il ne se déploie pas à partir de ce qui précède mon action sur l'objet. L'objet agit sur moi avant d'être compris par moi.

Et finalement, en cherchant tout ceci, nous nous pensons nous-même, nous pensons en même temps l'homme, ce penseur par excellence : toute interprétation présuppose une anthropologie et affecte l'anthropologie. Et si penser l'homme, c'est se penser soi-même comme un autre sans pouvoir être cet autre qui pense ou qui est pensé, est-ce seulement se penser par soi-même? L'homme peut penser par lui-même, il atteint même un certain résultat à le faire, une puissance accumulée au long des âges de son développement, et l'histoire de l'esprit humain a fait croire aux plus grands esprits à l'existence d'un progrès illimité du côté de l'homme, malheureusement asservi par une volonté de puissance. En revanche, lorsqu'il se pense lui-même, il ne peut pas se contenter de se penser par lui-même, car sinon il ne va nulle part, il produit un ensemble de tautologies ou d'impasses à son propos. L'anthropologie, à condition qu'elle veuille, non pas partir d'une définition simplement bumaine de l'homme, aujourd'hui d'un modèle ou d'un paradigme, comme le fait la sociologie, la médecine ou la biologie, peut parvenir à établir une définition de l'homme de sorte qu'il tende toujours un peu plus vers lui-même, pour l'aider à passer du sur-homme au plus-homme. C'est l'anthropologie qui naît de la théologie, et plus admirablement encore de la christologie?

\footnotetext{
${ }^{9}$ Cf. Vatican II, GS 22 : «En réalité, le mystère de l'homme ne s'éclaire vraiment que dans le mystère du Verbe incarné. Adam, en effet, le premier homme, était la figure de celui qui devait venir, le Christ Siegneur. Nouvel Adam, le Christ, dans la révélation même du mystère du Père et de son amour, manifeste pleinement l'homme à lui-même et lui découvre la sublimité de sa vocation. [...] «Image du Dieu invisible»(Col. 1, 15), il est l'Homme parfait qui a restauré dans la descendance d'Adam la ressemblance divine, altérée dès le premier péché. Parce qu'en lui la nature humaine a été assumée, non absorbée, par le fait même, cette nature a été élevée en nous aussi à une dignité sans égale.»
} 
On définit couramment l'anthropologie théologique comme la pensée de l'homme devant Dieu, ou pour mieux dire et éviter le sentiment d'être statique, la pensée de l'homme en devenir de Dieu. L'anthropologie théologique est devenue au XXe siècle une question majeure de la théologie : tournant anthropologique de la théologie ou plutôt tournant théologique de l'anthropologie, temps de réinvestissement d'une véritable théologie de l'homme, lui que l'on croyait définitivement coupé du Dieu que ceux que Nietzsche appelaient les épiciers avaient tués ${ }^{10}$. Cette nécessaire insertion de l'élément religieux dans la définition de l'homme est un enjeu majeur de notre temps. Quand je parle d'élément religieux, je ne parle pas seulement de l'homo religiosus qui est une donnée de fait : tout homme est religieux, ordonné vers ou tiraillé par un au-delà, par quelque horizon d'immortalité qu'on lui révèle, qu'il se fabrique, qu'il s'agisse de la surnature, de la politique ou de la technologie. Quand je parle de d'élément religieux, je pense à l'un des plus beaux enseignements de la Sainte Ecriture : l'imago Dei $i^{11}$.Que l'homme soit créé, soit, il ne s'est pas fait tout seul, nulle mère n'a jamais pensé sincèrement à sa démiurgie, même si beaucoup de mères peuvent être possessives, intrusives ou oppressives ${ }^{12}$. Elles donnent la vie, et pour ce faire doivent se donner tout court et ce geste d'offrande requiert vénération et gratitude ; mais en donnant la vie, en se donnant elles-mêmes, elles ne la créent pas. Que l'homme soit créé non à sa propre image, non à partir d'un premier exemplaire de lui-même est fascinant. Le prototype est Dieu lui-même, non comme le modèle de copies à venir (ratées en l'occurrence) mais comme la relation d'intimité et d'unicité qui permet à chacun de n'être la copie d'aucun prototype. La ressemblance n'est pas la pure identité, mais l'unité ou l'intimité ou la proximité, comme vous voudrez au seul être identique à lui-même, à l'unique, à l'Un, à l'Être, au Vivant, à l'Ipsum Esse subsistens, à celui que n'a jamais contenté d'être souverainement nécessaire, au Provident, au Dieu bon dont le psalmiste nous dit qu'il serait insensé de ne pas prendre sa promesse de salut au sérieux. L'homme est à son image et cette image est le Christ. Mais l'Archétype n'est pas une idée ou une valeur ou un standard, il est, et c'est un des grands paradoxes de notre foi, un chemin, un passage. En lui, Dieu et son plan de salut non seulement se font connaître, mais ils s'accomplissent, et, s'accomplissant, révèlent l'homme et à l'homme son salut comme recréation à l'image de Dieu, comme réhumanisation et divinisation dans le Christ.

La signification - ici Verbum caro factum est - entend porter le sens - ici Jésus de Nazareth jusqu'à nous. Est significatif le sens qui m'atteint bic et nunc - ici la sequela Christi. Est significatif ce qui a du poids pour moi, ce qui détermine ce vers quoi je tends - ici la visio beatifica - non pas seulement l'objet à désirer, mais l'élan que je prends ou le courant qui me traverse et me conduit vers lui. Et cet objet est vivant, et cet élan est vivant, et ce désir est vivant. La signification rompt la sphère immédiate de la vie humaine l'ouvrant à elle-même, la rendant présente à elle-même comme préoccupation et comme espérance. L'anglais meaning dit tellement plus. "It means to me » désigne ce qui nous anime tous et que nous appelons la "vérité », ce sens qui est donné à l'origine et découvert en vue de la fin, ce dévoilement sacré qui nous met face à elle in persona, le Crucifié-Ressuscité, qui l'identifie à nous, qui nous identifie à lui d'une manière dynamique et symphonique.

\section{III - L’homme en impératif de dignité}

\footnotetext{
${ }^{10}$ Cf. NietzsCHe, F., Ainsi parlait Zarathoustra (3e partie «En passant ») : « Ici pourissent tous les grands sentiments : on ne doit entendre ici que le craquement sec de petits sentiments secs et craquants. Ne sens-tu pas l'odeur des abattoirs des gargotes de l'esprit? Cette ville n'est-elle pas toute fumante des exhalaisons de l'esprit abattu ? Ne vois-tu pas les âmes accrochées comme des loques ramollies et sales? - et de ces loques, par-dessus le marché, ils font encore des journaux! N'entends-tu pas comme l'esprit est ici devenu un jeu de mots? Il dégorge une ignoble eau de vaisselle de mots? - et de cette eau de vaisselle de mots ils font encore des journaux! Ils s'excitent les uns les autres et ne savent pas vers quoi ? Ils s'échauffent les uns les autres et ne savent pas pourquoi ? Ils font retentir leur ferraille et sonner leur or. (...) Il y a aussi beaucoup de piété et beaucoup de pieux lécheurs de bottes, beaucoup de fabriquants de flatteries pour le Dieu des armées. (...) «Je sers, tu sers, nous servons », - voilà la prière que la vertu zélée fait monter vers le prince : pour que l'étoile méritée vienne enfin s'attacher à l'étroite poitrine ! (...) Le prince propose, mais l'épicier dispose !»

${ }^{11}$ Cf. Vatican II, GS 12 (et plus largement toute la partie I (L’Eglise et la vocation humaine) chapitre I (La dignité de la personne humaine) $\mathrm{n}^{\circ} 11-22$

12 Deus sive natura est la position d'un déisme immanentiste qui récuse le théisme judéo-chrétien ou l'existence d'un Etre créateur de toute chose, distinct de ses créatures. Nous lui préférons le mater sive natura.
} 
Pour le personnalisme chrétien, la liberté constitue le point nodal d'une définition de l'homme. Pas d'homme sans liberté, ni de dignité sans liberté. La liberté, et donc la dignité, commence par le fait de s'appartenir et cette auto-appartenance est un processus de libération et non une toute-puissance. S'appartenir, c'est justement se délester d'un programme de toute-puissance.

Le sens et le respect de la dignité personnelle est la marque d'une civilisation, civilisation au sens normatif ou éthique de ce qui s'oppose à la barbarie, donc notion universelle, c'est-à-dire necessaire et objective $^{13}$. Une société civilisée respecte la dignité humaine ou encore le droit (qui inclut de facto l'obligation et le besoin corollaires ${ }^{14}$ ) de tout homme d'atteindre son humanité. Respecte, c'est-à-dire non pas seulement «ne porte pas atteinte », mais aussi "protège et défend » les droits de la personne humaine, comme droits valant pour tout homme, quel qu'il soit, où qu'il soit : pour tous, toujours et partout. La liberté est le premier de ces droits. La condition d'esclave est le trait d'une société barbare, quelle que soit la hauteur de ses vues à propos de l'homme, puisqu'elle en vient à refuser à ceux qu'elle a soumis par les armes un autre statut que celui de butin ou de dommages de guerre payés par le vaincu $^{15}$. Pour comprendre quels sont ces droits fondamentaux, il faut comprendre ce qu'est la liberté qui les concrétise. Mais avant même de s'interroger sur ce qu'est la vraie liberté et sur le contenu d'une vraie liberté, il faut considérer que l'éthique n'est pas sans liberté même si cette liberté n'est pas encore éthique.

Ce primat de la liberté est impressionnant face à ce qui peut apparaître avec le libéralisme et l'individualisme actuels comme une liberté sans limite ${ }^{16}$. On peut défendre le primat de la liberté sans approfondir l'idée que la liberté n'est réelle qu'en étant morale, c'est-à-dire portée à l'accomplissement du bien. Mais pour ce faire, il faut une idée commune du bien, et pour ce faire, il faut une communauté. Ce n'est pas le bien commun qui fonde la communauté, c'est la communauté qui, existant concrètement, ne peut grandir et perdurer qu'en tendant vers un bien commun, qui dépasse non seulement l'intérêt de chacun de ses membres, mais encore son propre intérêt communautaire, car si la personne est une totalité ouverte, condition pour que le «nous » existe, il faut que le «nous » soit luimême une réalité sociale ouverte. Si la morale commune dépasse ma propre morale et guide ma propre action, la morale universelle dépasse la morale commune et guide l'action de la communauté1 ${ }^{17}$.

\footnotetext{
${ }^{13}$ Nous sommes conscients des limites de ces termes hors contexte et hors d'un cadre normatif supposé accepté par tous. Nous renvoyons ici à notre article «Culture et foi » dans Dictionnaire du Vatican et du Saint-Siège (dir. Christophe DicKES), Paris, Robert Laffont, Bouquins, p.355-358. Il existe trois conceptions de la culture : une conception classique ou humaniste («La» culture), la vision empirique ou sociologique («Toute» culture) et la vision locale ou particulariste («Telle» culture). Nous rapportons ces termes ici au contexte d'une culture humaniste.

14 On peut mettre les devoirs en simple regard des droits avec pour objet de limiter les seconds. Si les devoirs sont de simples fonctions (limitatrices) des droits, les ayant droit finiront par s'en défaire ou à les voir comme d'insupportables oppressions. Les droits engendrent des devoirs (envers autrui) parce qu'ils naissent des devoirs d'autrui envers moi. Mais pour que cela fonctionne, il faut que ces obligations réciproques soient fondées sur des besoins vitaux et leur prise de conscience. Sans autrui, je ne peux pas vivre, par conséquent, ma responsabilité est plus importante que mon droit à ceci ou cela. L'obligation n'est pas une limitation de mon droit, ou une opposition à lui, elle est une condition de possibilité et d'existence.

${ }^{15}$ Le droit de la guerre l'a toujours emporté sur les droits de l'homme, bien après la déclaration de ces droits imprescriptibles et universels. Il a ensuite été complété d'une justification naturaliste, estimant que certains peuples étaient esclaves par nature, et non plus seulement par défaite. On peut revenir sur une défaite, pas sur la nature. Le racialisme fut bien plus pérenne que la supériorité militaire pour justifier l'esclavage et que l'économie pour l'exiger.

${ }_{16}$ Pour Jacques Maritain, par exemple, libéralisme et totalitarisme sont les deux faces d'une même monnaie, l'un n'est pas l'antidote de l'autre.

${ }^{17}$ Le bien commun est la fin que se donne une société, parce qu’il est un principe d'unité qui dépasse les appartenances et les identités propres et parce que la société n'a pas pour fin le ou la somme des biens individuels de chacun. L'individualisme bourgeois est un anarchisme masqué : le libéralisme est dans cette zone grise où la liberté sert de paravent au droit des plus forts ou des plus riches. Le bien individuel promu pour lui-même absolument constitue à terme un droit d'opprimer celui qui n'a pas les moyens de se défendre. C'est le modèle dominant que l'Occident exporte et que nous voyons dans les démocraties libérales, et qui en vient même à les menacer aujourd'hui avec les mouvements populistes anti-démocratiques. Ce modèle confond le bien commun avec la «simple collection des biens privés "L'étatisme ou le collectivisme séparent complètement le bien commun et les biens individuels. Cf. MARITAIN, J., Christianisme et démocratie suivi de Les droits de l'homme, Paris, DDB, 1989, 2005 (2e édition), p.136.
} 
Pour le personnalisme chrétien, ensuite, définir l'homme comme «personne » signifie qu'on refuse de le réduire à un morceau de matière. L'individu délimite une entité, rationnelle dans le cas de l'homme, et la personne ouvre cette entité rationnelle qui devient une entité relationnelle. La dignité de l'homme est fondée sur son identité relationnelle, non pas seulement sur sa volonté, mais sur sa volonté en relation, et en relation au sens large, puisque l'homme n'est pas uniquement en relation avec ses semblables, mais avec tout ce qui constitue le cosmos, comme le rappelle l'écologie intégrale du pape François. Comme le dit Jacques Maritain, l'homme matériel existe, mais spirituellement il «sur-existe en connaissance et en amour ${ }^{18} \gg$. Si l'homme saisit au seul niveau de sa matérialité qu'il existe, il commence à vivre lorsqu'il est considéré au niveau de sa spiritualité.

Cette "sur-existence" est ce qui pour Maritain fonde une dignité absolue. Une dignité relative dépend de la réalisation de tel ou tel critère. Et chaque époque, chaque aire géographique et culturelle produit ses propres critères. Nous ne pouvons pas nous permettre d'adopter l'idée d'une dignité simplement relative appuyée sur tel standard économico-social ou culturel. Il faut que l'homme possède cette dignité en lui-même, et qu'il ne puisse jamais en être déchu. Il faut que l'homme soit substantiellement personne et non pas seulement juridiquement: la personnalité n'a pas à lui être accordée pour être, mais à être reconnue parce qu'il est. Sa simple presence suffit sans qu'il ait à revendiquer quoi que ce soit ou à passer quelque test de "personnalité". Sa personnalité n'est pas un concept simplement juridique, elle est un concept ontologique. Il ne peut donc pas exister d'être humain qui n'ait pas le statut de personne. Maritain ajoute que cette dignité absolue n'est pas l'absolu lui-même, mais, le « reflet d'un absolu supérieur au monde ${ }^{19}{ }$. Chacun n'est pas le tout, mais chacun est un tout.

Cette position, même si elle ne requiert pas d'avoir la foi en Jésus-Christ, est le fruit de la philosophie dite chrétienne, inscrite au cœur de la confession de foi, et non pas seulement en vue d'elle. Le personnalisme chrétien, que Maritain appelle «humanisme intégral» n'est pas le fruit d'une sécularisation des axiomes théologiques et religieux, mais une philosophie qui provient d'eux, qui saisit à la lumière de ces axiomes la valeur universelle de ce dont ils disposent. Il ne s'agit pas d'une universalité basée sur le dépassement du christianisme, mais d'une universalité produite par un christianisme confessant. L'axiome religieux, l'imago Dei est la racine sans laquelle tout arbre, si imposant soit-il, disparaît.

Alors, cet « homme intégral» ? Quel est-il sinon l'homme qui accueille sa vocation à être ? Si la personne est un tout, comme totalité spirituelle ouverte, elle n'est pas l'individu-roi ou le «petit dieu sans porte ni fenêtre », elle «tend », autrement dit elle vit son incomplétude non comme un drame mais comme un désir d'être, un désir de connaître, un désir de beauté, de vérité, d'unité, de bonté, comme une expérience de transcendance. Bernanos parlait de l'homme comme d'un pèlerin de l'absolu. Concrètement, ce désir, l'homme le vit à travers deux objets : la vie sociale et la communion. L'homme tend à vivre avec d'autres que lui et il tend à réaliser que la vie est communion, jusqu'à pouvoir s'offrir dans le don qui s'offre à lui et être un en lui et en tous par lui. Par la foi, il sait que ce don n'est pas seulement l'image du Dieu qui lui parle, mais que cette image du Dieu qui lui parle, et qui lui parle de Dieu, le représente aussi devant Dieu et devant les hommes comme sa propre image. L'homme a en lui une multitude d'images fausses de Dieu, de lui-même et d'autrui. L'image de Dieu révélée ne vient pas vaincre son ignorance, ou remplir un vide de représentations, mais sa méconnaissance, ou vaindre un trop plein de représentations, par l'aide il passera de l'idole à l'icône, et ainsi reconnaitra tous ses semblables jusqu'à s'y reconnaître par la mystérieuse présence de l'image en eux.

La dignité humaine est absolue, mais l'homme n'est pas absolu. Qu'est-ce à dire? Si la dignité personnelle de l'homme est absolue, sa liberté elle ne l'est pas, elle est relative à sa dignité. Au fond, il y a deux manières d'articuler les choses : soit la dignité de l'homme est relative à sa liberté absolue, soit la liberté de l'homme est relative à sa dignité absolue. Sa dignité est absolue au sens que rien ne vient la définir de l'extérieur: l'être humain est digne par nature, intrinsèquement. La dignité ne peut jamais l'abandonner. Sa dignité veut sa liberté, une liberté qui lui soit redevable et qui l'appelle à la gratitude, et

\footnotetext{
18 MARItAin, J., op.cit., p.132.
}

19 id., p.133. 
c'est parce que sa liberté est redevable à sa dignité que sa liberté est inaliénable et que la condition d'esclave est injustifiable et inacceptable, et que toute forme d'esclavage doit être combattu prioritairement, et que ce combat doit être considéré comme inséparable de la confession chrétienne.

Affirmer l'intégralité et l'intégrité de tout homme, l'inviolabilité de la personne humaine, ce n'est pas tout permettre à l'autonomie du sujet. La personne est limitée par sa propre inviolabilité. L'homme ne peut nier cette limite sans se mentir à lui-même ni se faire beaucoup de mal, sans s'oppresser luimême ou ses semblables ni détruire la "maison commune», parce que cette inviolabilité ou dignité absolue constituent le cadre de son autonomie. Il faudrait qu'il ait accès à ce qui le rend libre, qu'il ait totalement accès à son intériorité, ce qui n'est pas le cas. Il croit s'en sortir en délaissant son intériorité, mais le monde extérieur, si grand soit-il, est une prison, voire un bocal dans lequel il s'asphyxie. Le problème de la liberté n'est pas celui de la permissivité morale, mais celle du pouvoir, de la domination, de tout ce qui rend esclave de soi, du risque de l'auto-esclavage derrière la nécessité de l'autoaffirmation. L'obsession individuelle de la maitrise de soi, de sa vie, de son corps, de son temps, de ses envies, des autres produit des maitres sans disciple, sans transmission possible, sans communication réelle, sans amour durable. S’il n'y a pas de dignitas sans libertas, il n'y a pas de libertas sans auctoritas, sans le principe de croissance et d'excellence présent en nous comme la sève dans la plante. La liberté ne se limite pas à des libertés ou des droits revendiqués ou accordés, elle se fonde sur une expérience intérieure et spirituelle de transmission réciproque et de croissance mutuelle.

Et nous voici revenus au terminus quo de notre intervention : la transmission de ce qui a été reçu, et sa comprehension par la recherche en vue de sa communication sous la forme d'un message significatif. La significativité du christianisme ne repose pas seulement sur la crédibilité rationnelle de ce qu'il dit, elle s'enracine dans l'identité du disciple qui le reste toujours, surtout lorsqu'il embrasse le devoir de chercher, d'enseigner et de témoigner. 